\title{
Spin-half paramagnetism in graphene induced by point defects
}

\author{
R. R. Nair ${ }^{1}$, M. Sepioni ${ }^{1}$, I-Ling Tsai ${ }^{1}$, O. Lehtinen ${ }^{2}$, J. Keinonen ${ }^{2}$, A. V. Krasheninnikov ${ }^{2,3}$, T. Thomson ${ }^{1}$, \\ A. K. Geim ${ }^{1}$ and I. V. Grigorieva ${ }^{1 \star}$
}

\begin{abstract}
The possibility to induce a magnetic response in graphene by the introduction of defects has been generating much interest, as this would expand the already impressive list of its special properties and allow novel devices where charge and spin manipulation could be combined. So far there have been many theoretical studies (for reviews, see refs 1-3) predicting that point defects in graphene should carry magnetic moments $\mu \sim \mu_{\mathrm{B}}$ and these can in principle couple (anti)ferromagnetically ${ }^{1-12}$. However, experimental evidence for such magnetism remains both scarce and controversial $\left.\right|^{13-16}$. Here we show that point defects in graphene-(1) fluorine adatoms in concentrations $x$ gradually increasing to stoichiometric fluorographene $\mathbf{C F}_{\mathbf{x}=1.0}$ (ref. 17) and (2) irradiation defects (vacancies)-carry magnetic moments with spin 1/2. Both types of defect lead to notable paramagnetism but no magnetic ordering could be detected down to liquid helium temperatures. The induced paramagnetism dominates graphene's low-temperature magnetic properties, despite the fact that the maximum response we could achieve was limited to one moment per approximately 1,000 carbon atoms. This limitation is explained by clustering of adatoms and, for the case of vacancies, by the loss of graphene's structural stability. Our work clarifies the controversial issue of graphene's magnetism and sets limits for other graphitic compounds.
\end{abstract}

The emerging consensus that magnetism in carbon-based systems can exist is based mostly on a large body of work on magnetic measurements of highly-oriented pyrolytic graphite (HOPG) and carbon films, with many reports of weak ferromagnetic signals at room temperature $(T)$ observed in both pristine HOPG and after its ion irradiation (see, for example, refs 18,19 ). However, the whole subject remains controversial, especially concerning (1) the role of possible contamination and (2) the mechanism responsible for the strong interaction required to lead to ferromagnetic ordering at room temperature. Some observations of ferromagnetism are probably artefacts, doing little justice to the subject (one frequent artefact is identified and described in the Supplementary Information, where we show that commonly used HOPG crystals contain micrometre-sized magnetic particles). Adatom magnetism in graphite is also contentious and, for example, different studies of fluorinated graphite have reported inconsistent results ${ }^{20,21}$.

Graphene, as the basic building block of all graphitic materials, is simpler than its three-dimensional (3D) counterparts and can be used to address questions that are harder to address in 3D materials with complex electronic spectra. Since sufficient quantities of graphene became available in the form of laminates (large collections of electronically non-interacting graphene crystallites ${ }^{15,22}$ ), it has become possible to use SQUID magnetometry to show that pristine graphene is strongly diamagnetic and shows only a tiny background paramagnetism that requires $T<50 \mathrm{~K}$ to be noticeable ${ }^{15}$. As concerns the role of defects, the most relevant observations so far are probably the report of spin-1 paramagnetism in ion-implanted graphitic nanoflakes ${ }^{16}$ and the Kondo effect and giant negative magnetoresistance in defected-graphene devices ${ }^{23,24}$, although the former disagrees with the spin $1 / 2$ universally expected for point defects ${ }^{1-12}$ and the latter are transport experiments allowing different interpretations ${ }^{12,24}$. Let us also mention the observation of a peak in the tunnelling density of states near vacancies, even though that work was carried out for graphite ${ }^{25}$.

In this report, we have employed the above graphene laminates as a well-characterized and clean reference system to study the effect of the controlled introduction of point defects on graphene's magnetic properties. Starting samples were prepared by ultrasonic cleavage of high-purity HOPG in an organic solvent, $\mathrm{N}$-methylpyrrolidone, following the procedure described in ref. 15 and Supplementary Information. The resulting laminates (Fig. 1a) consist of 10-50 $\mathrm{nm}$ graphene crystallites, predominantly monoand bilayers, aligned parallel to each other and electronically decoupled. The magnetization behaviour of the pristine laminates has been described in detail in our previous study ${ }^{15}$. Briefly, it shows a purely diamagnetic response over a wide range of $T$. Weak background paramagnetism becomes noticeable only below $50 \mathrm{~K}$ and corresponds to a low density $(\sim 40 \mathrm{ppm})$ of magnetic moments, with approximately one spin per graphene crystallite. The origin of this paramagnetism remains unclear but is unlikely to be due to remnant magnetic impurities or graphene's edge states and point defects ${ }^{15}$.

To achieve a gradually increasing concentration of fluorine atoms attached to graphene sheets, we followed the procedures of ref. 17, namely, a whole sample such as that shown in Fig. 1a was placed in a Teflon container and, for a number of hours, exposed to atomic $\mathrm{F}$ formed by the decomposition of xenon difluoride at $200{ }^{\circ} \mathrm{C}$. During fluorination the graphene was in contact only with $\mathrm{XeF}_{2}$ and Teflon, and test measurements (heating a sample in the same container but without $\mathrm{XeF}_{2}$ ) showed that no contamination was introduced during preparation. A progressive increase in the F/C ratio was monitored by Raman spectroscopy (Supplementary Information), as well as the colour change: from metallic dark grey, through brown to light yellow-see Fig. 1b. For quantitative analysis we used X-ray photoemission spectroscopy, which allowed us to measure the value of $x$ in $\mathrm{CF}_{x}$ (for example $\mathrm{CF}_{x=0.1}$ corresponds to an $\mathrm{F} / \mathrm{C}$ ratio of 0.1 ). Details of the composition analysis are given in the Supplementary Information.

The exposure to atomic fluorine resulted in strong paramagnetism, such that the low- $T$ saturation magnetization increased by

${ }^{1}$ Manchester Centre for Mesoscience and Nanotechnology, Manchester M13 9PL, UK, ${ }^{2}$ Department of Physics, University of Helsinki, PO Box 43, FI-00014, Finland, ${ }^{3}$ Department of Applied Physics, Aalto University, PO Box 11100, Fl-00076, Finland. *e-mail: irina.grigorieva@manchester.ac.uk. 

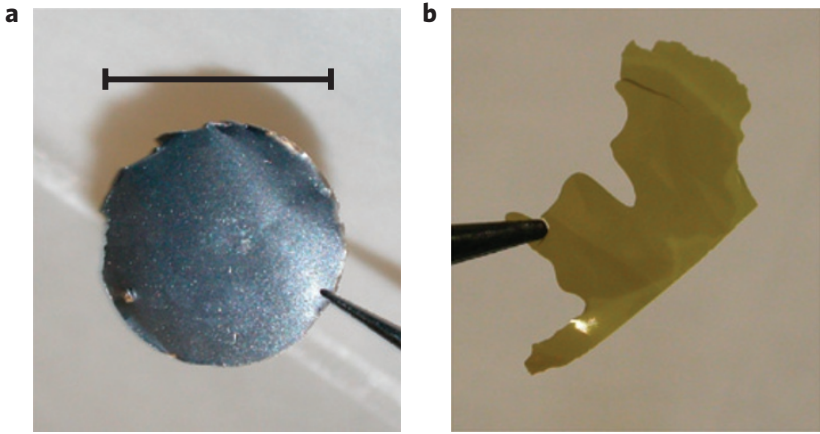

Figure $\mathbf{1}$ | Experimental samples. a,b, Optical images of starting (a) and fully fluorinated (b) graphene laminates. The scale bar is $2 \mathrm{~cm}$. The laminates consist of 10-50 nm graphene crystallites, predominantly monoand bilayers ${ }^{15}$, aligned parallel to each other (as seen in a scanning electron microscope), rotationally disordered and, consequently, electronically decoupled ${ }^{22}$

more than an order of magnitude with respect to the background signal in the initial samples. Figure 2 shows the evolution of the magnetization in the $\mathrm{CF}_{x}$ samples with $x$ increasing from 0.1 to 1 (fluorographene). At all fluorine concentrations the behaviour is accurately described by the Brillouin function

$$
M=N g J \mu_{\mathrm{B}}\left[\frac{2 J+1}{2 J} \operatorname{ctnh}\left(\frac{(2 J+1) z}{2 J}\right)-\frac{1}{2 J} \operatorname{ctnh}\left(\frac{z}{2 J}\right)\right]
$$

where $z=g J \mu_{\mathrm{B}} H / k_{\mathrm{B}} T, g$ is the $g$-factor, $J$ the angular momentum number, $N$ the number of spins and $k_{\mathrm{B}}$ the Boltzmann constant. The Brillouin function provides good fits only for $J=S=1 / 2$ (free electron spin). Other $J$ unequivocally disagree with the functional form of the measured $M(H)$, as they give qualitatively different, sharper changes with faster saturation (for details of defining $J$, see Supplementary Information). This behaviour is corroborated by fits of $M(T)$ to the Curie law curves $\chi=M / H=N J(J+1) g^{2} \mu_{\mathrm{B}}^{2} /\left(3 k_{\mathrm{B}} T\right)$, which were calculated for $J=1 / 2$ and $N$ inferred from the $M(H)$ curves-see Fig. 2b. Given the many reports of defect-induced ferromagnetism in carbon systems, we paid particular attention to any signs of magnetic ordering in our samples. No signatures were found with accuracy better than $10^{-5}$ e.m.u. $\mathrm{g}^{-1}$ - see inset in Fig. $2 \mathrm{~b}$.

The evolution of the number of spins $N$, extracted from the measured $M(H, T)$, with an increasing degree of fluorination is shown in Fig. 3. $N$ increases monotonically with $x$ up to $x \approx 0.9$, then shows some decrease for the fully fluorinated samples. It is instructive to replot the same data in terms of the number of Bohr magnetons, $\mu_{\mathrm{B}}$, per attached $\mathrm{F}$ atom (inset in Fig. 3). It is clear that the initial increase (up to $x \approx 0.5$ ) in the number of paramagnetic centres is proportional to $x$, as expected, but for higher $x$ the behaviour reveals a more complicated relation between the number of adatoms and $N$. Furthermore, for all $x$ the measured number of paramagnetic centres is three orders of magnitude less than the measured number of $\mathrm{F}$ adatoms in the samples, that is, only one out of $\sim 1,000$ adatoms seems to contribute to the paramagnetism. This may seem surprising because the general expectation is that each adatom should contribute $\sim \mu_{\mathrm{B}}$ to the total $M$ (refs 1-11). However, we recall that fluorine atoms on graphene have a strong tendency towards clustering because of low migration barriers ${ }^{26-28}$. The tendency towards clustering is further enhanced by the presence of corrugations (ripples) ${ }^{26,28}$. The value of $\mu_{\mathrm{B}} / \mathrm{F}_{\text {_atom of }} \sim 10^{-3}$ implies clusters of $\sim 8 \mathrm{~nm}$ in size, which agrees with the measured typical sizes of ripples in graphene ${ }^{29}$. In the case of clustering, the magnetic moment from the interior of a cluster is expected to be zero because of the bipartite nature of the graphene

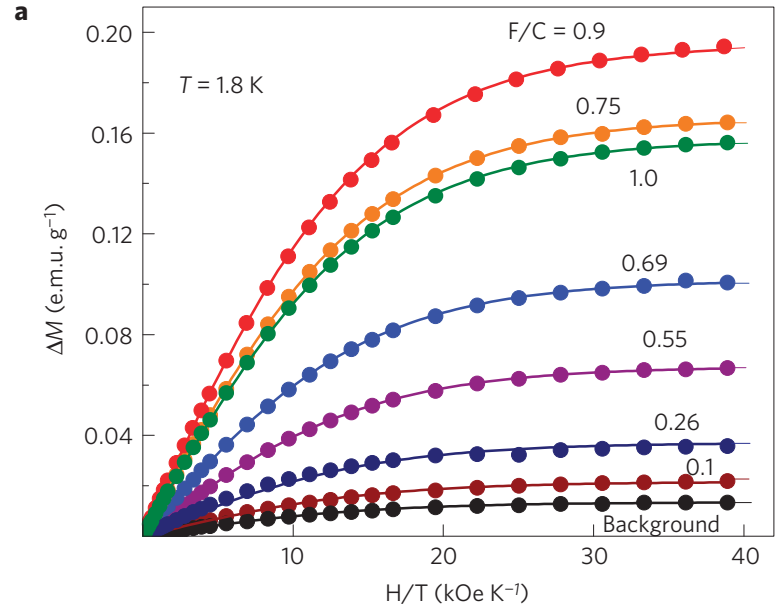

b

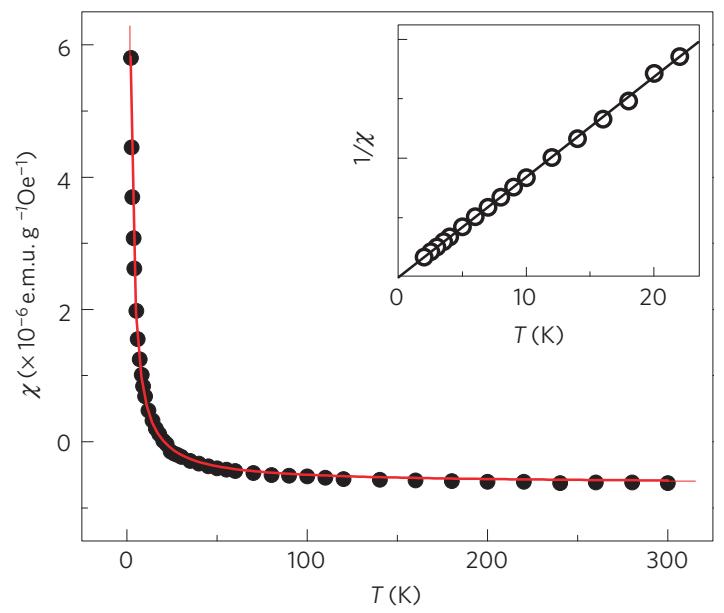

Figure 2 | Paramagnetism due to fluorine adatoms. a, Magnetic moment $\Delta M$ (after subtracting linear diamagnetic background) as a function of parallel field $H$ for different $F / C$ ratios. Symbols are the measurements and solid curves are fits to the Brillouin function with $S=1 / 2$ and assuming $g=2$ (the fits weakly depend on $g$ (ref. 15)). b, Example of the dependence of susceptibility $\chi=M / H$ on $T$ in parallel $H=3 \mathrm{kOe}$ for $\mathrm{CF}_{x}$ with $x=0.9$; symbols are the measurements and the solid curve is the Curie law calculated self-consistently using the $\mathrm{M} / \mathrm{H}$ dependence found in $\mathbf{a}$. Inset: Inverse susceptibility versus $T$ demonstrating a linear, purely paramagnetic behaviour with no sign of magnetic ordering.

lattice ${ }^{1,3,30}$. Therefore, a magnetic contribution can come only from cluster edges and would be determined by a particular configuration of adatoms near the edges, that is, only those adatoms on the A sublattice that have no counterparts on the neighbouring sites of the B sublattice will contribute to $M$. Accordingly, each cluster is expected to contribute only a few $\mu_{\mathrm{B}}$ to the total magnetization, which could explain our observations.

As $x$ increases, it is reasonable to expect that at some point all strongly curved areas of the graphene sheet become occupied with fully formed $\mathrm{F}$ clusters so that there appears an increasing number of isolated (that is, magnetic) adatoms or small clusters. Their contribution can explain the observed nonlinear increase in $\mu_{\mathrm{B}}$ per adatom for $x>0.5$. As $\mathrm{CF}_{x}$ approaches the stoichiometric compound $(x=1)$, we observe a notable $(\sim 20 \%)$ decrease in $M$ as compared with $x \approx 0.9$ (confirmed in several independent measurements) but the material remains strongly paramagnetic. The latter is somewhat surprising, because stoichiometric fluorographene should probably ${ }^{11}$ be nonmagnetic. However, even for $x=0.999$, there are still a large number of defects present in the fluorographene lattice $(\sim$ one missing $\mathrm{F}$ atom 


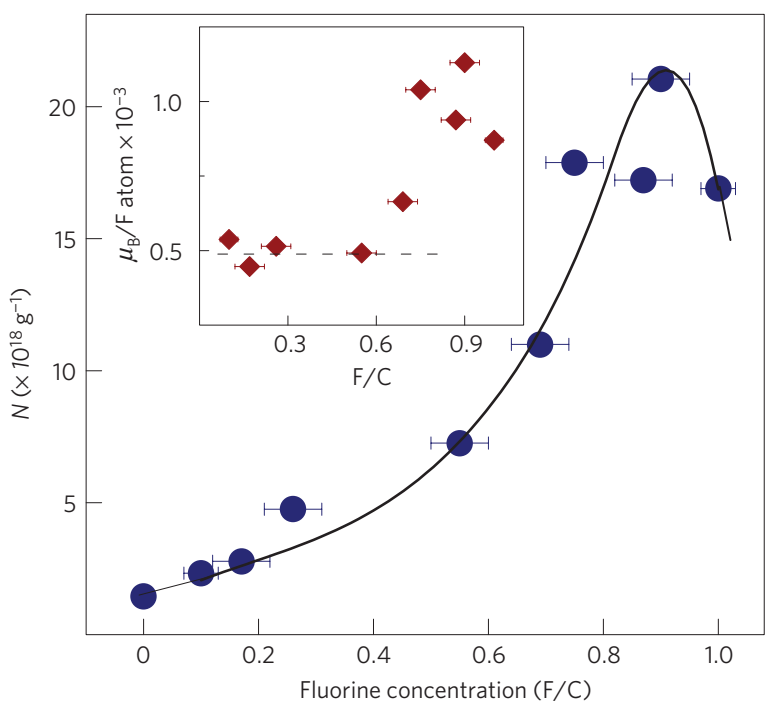

Figure 3 | Adatom paramagnetism. Main panel: Number of spins $N$ extracted from the Brillouin fits in Fig. 2 as a function of $F / C$ ratio. The solid curve is a guide to the eye. Inset: the same $N$ normalized to the concentration of adatoms in each sample ( $\mu_{\mathrm{B}} / \mathrm{F}$ atom is obtained by dividing the number of moments $N$, assuming that each carries $1 \mu_{\mathrm{B}}$, by the number of $\mathrm{F}$ atoms per $\mathrm{g}$ of fluorinated graphene). Error bars indicate the accuracy of determination of the fluorine concentrations.

per 1,000) and we speculate that the resulting midgap states associated with such defects and found in fluorographene ${ }^{17}$ can have a magnetic moment, similar to the predicted behaviour of $\mathrm{H}$-vacancies in graphane $\mathrm{e}^{11}$.

To explore the generality of the magnetic behaviour described above, we investigated the magnetic response associated with another type of point defect, namely, vacancies produced by the irradiation of graphene with high-energy protons and carbon $\left(\mathrm{C}^{4+}\right)$ ions. Unlike $\mathrm{F}$ atoms, vacancies are not mobile at room temperature and cannot form clusters, but otherwise their contribution to magnetism is expected to be similar to that of adatoms (both types of defect lead to the formation of localized states at energies corresponding to the vanishing density of states ${ }^{1-12}$ and are often referred to as $p_{z}$-vacancies $\left.{ }^{1}\right)$. Also, note that interstitial carbon atoms created along with the vacancies are not expected to carry a magnetic moment ${ }^{31}$.

For our irradiation experiments, we started with the same graphene laminates as used for fluorination. The energy, fluence and other irradiation parameters for each sample were chosen on the basis of computer simulations (SRIM software package) to achieve a desired defect density and ensure a uniform defect distribution. The ion energies-350-400 keV for protons and $20 \mathrm{MeV}$ for $\mathrm{C}^{4+}$ — were chosen to ensure that the stopping ranges for the $\mathrm{H}^{+}$and $\mathrm{C}^{4+}$ ions exceeded the sample thicknesses, thus leaving behind only irradiation-induced defects but no implanted ions. During the irradiation, special care was taken to prevent sample contamination and to avoid any significant vacancy-interstitial recombination by ensuring that the sample temperature remained below $50^{\circ} \mathrm{C}$. The total number of vacancies in each sample was estimated by means of SRIM simulations. Further details are given in the Supplementary Information.

The results of magnetization measurements for vacancies are summarized in Fig. 4. For all concentrations, $M(H)$ curves, such as those shown in the inset, correspond accurately to paramagnetism of non-interacting spins with $S=1 / 2$ (solid curves are fits to the Brillouin function). This result disagrees with ref. 16 but is in agreement with theory and directly proves the case of the much-discussed magnetism due to vacancies in graphene $e^{1-12}$.

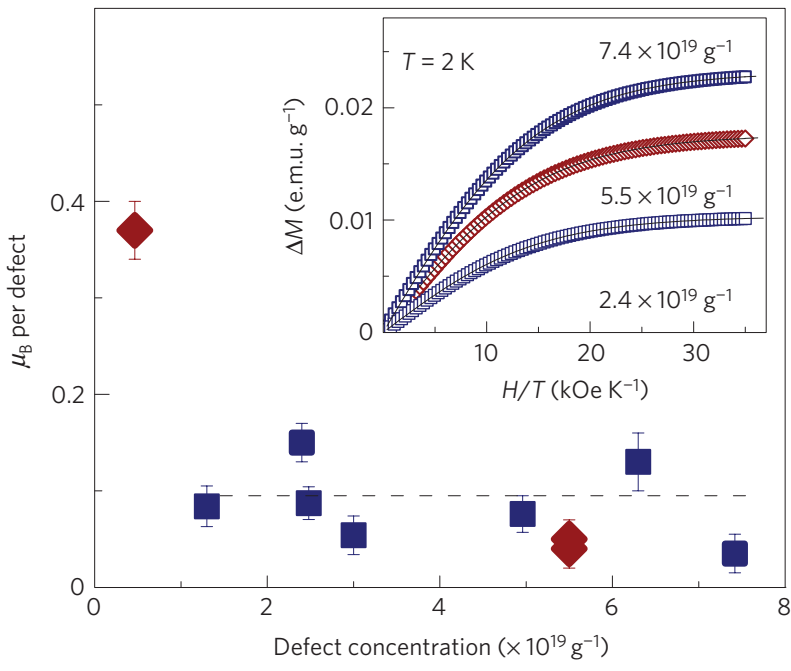

Figure 4 | Vacancy paramagnetism. Magnetic properties of graphene laminates irradiated with protons (blue squares) and $\mathrm{C}^{4+}$ ions (red diamonds). Main panel: Magnetic moment $M$ normalized by the concentration of vacancies. Error bars indicate the accuracy of determination of the number of spins per vacancy. Inset: Magnetic moment $\Delta M$ due to vacancies as a function of parallel field $H$. The labels give the defect density; solid curves are Brillouin function fits for $J=1 / 2$.

Also, it is clear from Fig. 4 that irradiation defects give rise to the same paramagnetism, independent of the ions used $\left(\mathrm{H}^{+}\right.$or $\mathrm{C}^{4+}$ ), and there is no sign of ferromagnetic ordering (also, see Supplementary Information).

At first glance, the effect of irradiation is rather similar to that of fluorination. However, the maximum total increase in $M$ that we could achieve in the irradiation experiments was $\sim 10$ times smaller than the maximum $M$ achieved with adatoms (see Fig. 2). This is simply because we could only introduce a limited number of vacancies and, for defect densities above $10^{20} \mathrm{~g}^{-1}$, our samples became so fragile that they disintegrated. Despite the smaller achieved magnetization, the effect of individual vacancies is much larger than in the case of $\mathrm{F}$ adatoms. Figure 4 shows the magnetic moment per vacancy, calculated from the $M(H)$ curves, versus the estimated density of vacancies in each of the studied samples. The values of $\mu$ found lie between 0.1 and $0.4 \mu_{\mathrm{B}}$ per vacancy, which should be considered in reasonable agreement with the theoretical value of $\mu \sim \mu_{\mathrm{B}}$ expected for isolated vacancies ${ }^{1-12}$. Indeed, there are considerable uncertainties in estimating the number of vacancies in our samples. First, SRIM simulations work well for graphite but cannot take into account the exact crystallography of graphene laminates and the varying separations between crystallites. More importantly, there is a possibility of reconstruction of individual vacancies into, for example, double vacancies, chemically terminated dangling bonds, Stone-Wales defects and so on, which are expected to be nonmagnetic ${ }^{10}$. Furthermore, the possibility of vacancy-interstitial recombination during or after irradiation cannot be ruled out completely (see Supplementary Information).

To conclude, the observation of magnetic vacancies and adatoms with spin $1 / 2$ provides most unambiguous direct support for many theories discussing graphene's magnetic properties. As for the intensely debated ferromagnetism in graphitic compounds, no magnetic ordering was detected, with both fluorinated and irradiated samples exhibiting purely paramagnetic behaviour even for the largest defect densities at the lowest $T$ of $2 \mathrm{~K}$. This agrees with straightforward expectations. Indeed, the concentration of magnetic moments we could achieve was only $\sim 0.1 \%$ of the maximum hypothetically possible magnetism of one moment per carbon atom. In the case of fluorine adatoms, this relative 
inefficiency of introducing paramagnetic centres is explained by clustering, such that neighbouring adatoms residing on different graphene sublattices do not contribute to the overall magnetic moment. For vacancies, their density was limited by the requirement to retain the structural integrity of graphene. Although the achieved concentrations of paramagnetic centres are notably higher than the ferromagnetic fractions typically reported for graphitic materials, the average spacing between magnetic moments is still rather large $\sim 10 \mathrm{~nm}$, apparently too large for magnetic ordering even at liquid helium temperatures.

Received 22 September 2011; accepted 24 November 2011; published online 10 January 2012

\section{References}

1. Yazyev, O. V. Emergence of magnetism in graphene materials and nanostructures. Rep. Prog. Phys. 73, 056501 (2010).

2. Castro Neto, A. H. et al. Adatoms in graphene. Solid State Commun. 149, 1094-1100 (2009).

3. Wehling, T., Katsnelson, M. \& Lichtenstein, A. Adsorbates on graphene: Impurity states and electron scattering. Chem. Phys. Lett. 476, 125-134 (2009).

4. Yazyev, O. V. \& Helm, L. Defect-induced magnetism in graphene. Phys. Rev. B 75, 125408 (2007).

5. Kumazaki, H. \& Hirashima, D. S. Nonmagnetic-defect-induced magnetism in graphene. J. Phys. Soc. Jpn 76, 064713 (2007).

6. Uchoa, B., Kotov, V. N., Peres, N. M. R. \& Castro Neto, A. H. Localized magnetic states in graphene. Phys. Rev. Lett. 101, 026805 (2008).

7. Palacios, J. J., Fernández-Rossier, J. \& Brey, L. Vacancy-induced magnetism in graphene and graphene ribbons. Phys. Rev. B 77, 195428 (2008).

8. Singh, R. \& Kroll, P. Magnetism in graphene due to single-atom defects: Dependence on the concentration and packing geometry of defects. J. Phys. Condens. Matter 21, 196002 (2009).

9. Li, W., Zhao, M., Xia, Y., Zhang, R. \& Mu, Y. Covalent-adsorption induced magnetism in graphene. J. Mater. Chem. 19, 9274-9282 (2009).

10. López-Sancho, M. P., de Juan, F. \& Vozmediano, M. A. H. Magnetic moments in the presence of topological defects in graphene. Phys. Rev. B 79, 075413 (2009).

11. Berashevich, J. \& Chakraborty, T. Sustained ferromagnetism induced by H-vacancies in graphane. Nanotechnology 21, 355201 (2010).

12. Haase, P., Fuchs, S., Pruschke, T., Ochoa, H. \& Guinea, P. Magnetic moments and Kondo effect near vacancies and resonant scatterers in graphene. Phys. Rev. B 83, 241408 (2011).

13. Wang, Y. et al. Room-temperature ferromagnetism of graphene. Nano Lett. $\mathbf{9}$, 220-224 (2009).

14. Ramakrishna Matte, H. S. S., Subrahmanyam, K. S. \& Rao, C. N. R. Novel magnetic properties of graphene: Presence of both ferromagnetic and antiferromagnetic features and other aspects. J. Phys. Chem. C 113, 9982-9985 (2009).

15. Sepioni, M. et al. Limits on intrinsic magnetism in graphene. Phys. Rev. Lett. 105, 207205 (2010).
16. Ney, A., Papakonstantinou, P., Kumar, A., Shang, N. G. \& Peng, N. Irradiation enhanced paramagnetism on graphene nanoflakes. Appl. Phys. Lett. 99, 102504 (2011).

17. Nair, R. R. et al. Fluorographene: A two dimensional counterpart of Teflon. Small 6, 2877-2884 (2010).

18. Esquinazi, P. et al. Induced magnetic ordering by proton irradiation in graphite. Phys. Rev. Lett. 91, 227201 (2003).

19. Makarova, T. L., Shelankov, A. L., Serenkov, I. T., Sakharov, V. I. \& Boukhvalov, D. W. Anisotropic magnetism of graphite irradiated with medium-energy hydrogen and helium ions. Phys. Rev. B 83, 085417 (2011).

20. Panich, M., Shamesa, A. I. \& Nakajimab, T. On paramagnetism in fluorinated graphite: EPR and solid state NMR study. J. Phys. Chem. Solids 62, 959-964 (2001).

21. Takai, K. et al. Effect of fluorination on nano-sized $\pi$-electron systems. J. Phys. Soc. Jpn 70, 175-185 (2001).

22. Luican, A. et al. Single-layer behavior and its breakdown in twisted graphene layers. Phys. Rev. Lett. 106, 126802 (2011).

23. Chen, J-H., Li, L., Cullen, W. G., Williams, E. D. \& Fuhrer, M. S. Tunable Kondo effect in graphene with defects. Nature Phys. 7, 535-538 (2011).

24. Hong, X., Cheng, S. H., Herding, C. \& Zhu, J. Colossal negative magnetoresistance in dilute fluorinated graphene. Phys. Rev. B 83, 085410 (2011).

25. Ugeda, M. M., Brihuega, I., Guinea, F. \& Rodri'guez, M.G. Missing atom as a source of carbon magnetism. Phys. Rev. Lett. 104, 096804 (2010).

26. Osuna, S. et al. Reaction mechanisms for graphene and carbon nanotube fluorination. J. Phys. Chem. C 114, 3340-3345 (2010).

27. Şahin, H., Topsakal, M. \& Ciraci, S. Structures of fluorinated graphene and their signatures. Phys. Rev. B 83, 115432 (2011).

28. Boukhvalov, D. \& Katsnelson, M. Enhancement of chemical activity in corrugated graphene. J. Phys. Chem. C 113, 14176-14178 (2009).

29. Meyer, C. et al. The structure of suspended graphene sheets. Nature 446, 60-63 (2007)

30. Rappoport, T. G., Uchoa, B. \& Castro Neto, A. H. Magnetism and magnetotransport in disordered graphene. Phys. Rev. B 80, 245408 (2009).

31. Gulans, A., Krasheninnikov, A. V., Puska, M. J. \& Nieminen, R. M. Bound and free self-interstitial defects in graphite and bilayer graphene: A computational study. Phys. Rev. B 84, 024114 (2011).

\section{Acknowledgements}

This work was supported by the UK Engineering and Physical Sciences Research Council and by the Academy of Finland.

\section{Author contributions}

I.V.G. and A.K.G. devised the project, analysed the results and wrote the paper. I.V.G. supervised the experiments. R.R.N. carried out measurements and analysis of fluorinated graphene. M.S. and I-L.T. carried out magnetization measurements of ion-irradiated graphene. M.S. carried out measurements of HOPG. O.L., J.K. and A.V.K. carried out ion irradiations and analysis of defect structures. All authors contributed to discussions.

\section{Additional information}

The authors declare no competing financial interests. Supplementary information accompanies this paper on www.nature.com/naturephysics. Reprints and permissions information is available online at www.nature.com/reprints. Correspondence and requests for materials should be addressed to I.V.G. 